CRYSTALLOGRAPHIC COMMUNICATIONS

ISSN 2056-9890

Received 6 April 2018

Accepted 24 April 2018

Edited by J. Ellena, Universidade de Sâo Paulo, Brazil

Keywords: azopyrrole; borondifluoride complex; crystal structure; hydrogen bond.

CCDC reference: 1839158

Supporting information: this article has supporting information at journals.iucr.org/e

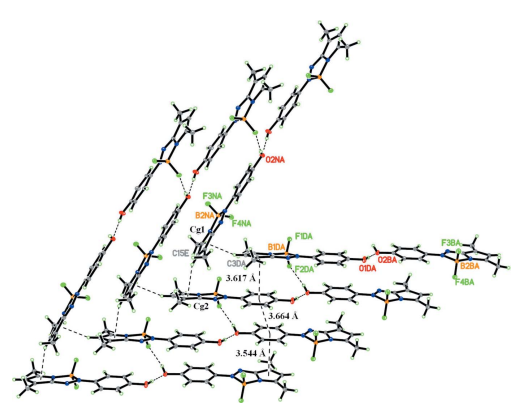

OPEN $\odot$ ACCESS

\section{Crystal structure of difluorido\{2-[(4-hydroxyphen- yl)diazenyl]-3,5-dimethylpyrrolido\}boron}

\author{
Huixiao Feng ${ }^{\mathrm{a}, \mathrm{b}}$ and Zhenming Yin ${ }^{\mathrm{a}, \mathrm{b} *}$ \\ ${ }^{a}$ College of Chemistry, Tianjin Key Laboratory of Structure and Performance for Functional Molecules, Tianjin Normal \\ University, Tianjin 300387, People's Republic of China, and ${ }^{\mathbf{b}}$ Key Laboratory of Inorganic-Organic Hybrid Functional \\ Materials Chemistry, (Tianjin Normal University), Ministry of Education, Tianjin 300387, People's Republic of China. \\ *Correspondence e-mail: tjyinzm@aliyun.com
}

The asymmetric unit of the title azopyrrole- $\mathrm{BF}_{2}$ complex, $\mathrm{C}_{12} \mathrm{H}_{12} \mathrm{BF}_{2} \mathrm{~N}_{3} \mathrm{O}$, contains two independent molecules, which are linked by an $\mathrm{O}-\mathrm{H} \cdots \mathrm{O}$ hydrogen bond. The dimers are further assembled into a one-dimensional ladder-like structure through $\mathrm{O}-\mathrm{H} \cdots \mathrm{F}$ hydrogen bonds and stabilized by $\pi-\pi$ interactions. The ladders are further linked by $\mathrm{C}-\mathrm{H} \cdots \pi$ contacts.

\section{Chemical context}

Recently, some unique pyrrole- $\mathrm{BF}_{2}$-based dyes have emerged as alternatives to 4,4-difluoro-4-bora-3a,4a-diaza-s-indacene (BODIPY) dyes because of their easy synthesis, lower symmetry and longer wavelengt absorption. Li et al. (2009) have synthesized a series of azopyrroles and their difluoroboron complexes, which possess promising absorption properties. The potentials of a few $\mathrm{BF}_{2}$-azopyrrole complexes as sensitizers for dye-sensitized solar cells (DSSCs) have been evaluated (Mikroyannidis, Royd et al., 2010). In the meantime, some $\mathrm{BF}_{2}$-azopyrrole complexes have been used for the fabrication of bulk heterojunction solar cells (Mikroyannidis, Kabanakis et al., 2010). A 2-(dimethylaminophenylazo)-5ethyl-pyrrole boron difluoride complex has been used as an OFF-ON-OFF-type three-stage binary $\mathrm{pH}$ switch (Lee et al., 2012). Previously, we have reported the crystal structures of some azopyrrole compounds (Yin et al., 2008; Li et al., 2011). In an extension of this research, we report herein on the crystal structure of difluorido\{2-[(4-hydroxyphenyl)diazenyl]3,5-dimethylpyrrolido\}boron.

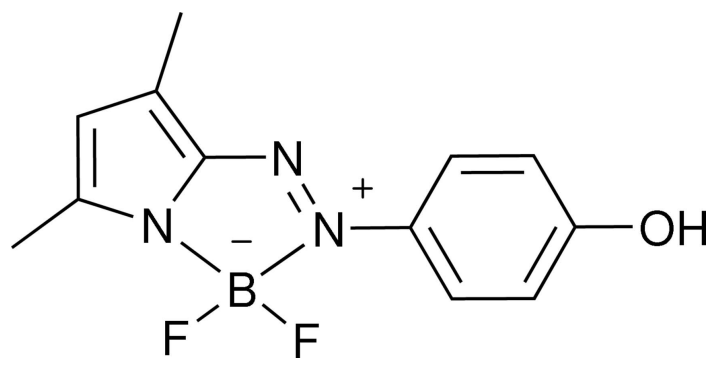

\section{Structural commentary}

The asymmetric unit contains two independent molecules, which show slight differences in some bond lengths [e.g. O1$\mathrm{C} 10$ and $\mathrm{O} 2-\mathrm{C} 22=1.358(3)$ and $1.382(3) \AA$, respectively; 


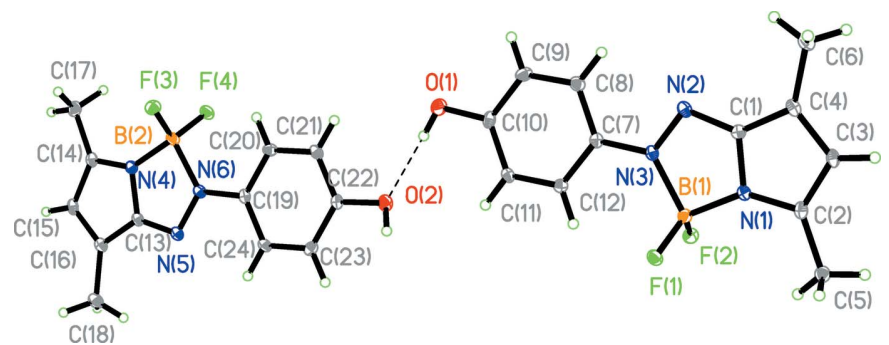

Figure 1

The asymmetric unit of the title compound, with displacement ellipsoids drawn at the $30 \%$ probability level. The $\mathrm{O}-\mathrm{H} \cdots \mathrm{O}$ hydrogen bond is shown as a dashed line.

Table 1] and torsion angles [N2-N3-C7- 12 and $\mathrm{N} 5-$ $\mathrm{N} 6-\mathrm{C} 19-\mathrm{C} 20$ are $-171.1(2)$ and $177.9(2)^{\circ}$, respectively]. The r.m.s. deviation for fitting two molecules $=0.055 \AA$. The two molecules are linked by the $\mathrm{O} 1-\mathrm{H} 1 \cdots \mathrm{O} 2$ hydrogen bond (Fig. 1, Table 2). The torsion angles between benzene rings and neighboring pyrrole rings in the N1- and N4-containing molecules are $9.43(12)$ and $1.34(12)^{\circ}$, respectively. Each boron atom is four-coordinated by two fluorine atoms, a pyrrole $\mathrm{N}$ atom and an azo $\mathrm{N}$ atom. The $\mathrm{B}-\mathrm{N}$ bond distances vary from 1.537 (3) to 1.618 (3) $\AA$ (Table 1). The $\mathrm{B}-\mathrm{N}_{\text {pyrrole }}$ bonds are shorter than the $\mathrm{B}-\mathrm{N}_{\mathrm{azo}}$ bonds. The two $\mathrm{N}-\mathrm{N}$ bonds each adopt a trans conformation and at 1.318 (3) and 1.312 (3) $\AA$ are much longer than that in the structure of the free azopyrrole ligand (Yin et al., 2008). In addition, the C1-
Table 1

Selected bond lengths (@).

\begin{tabular}{llll}
\hline $\mathrm{F} 1-\mathrm{B} 1$ & $1.369(3)$ & $\mathrm{F} 3-\mathrm{B} 2$ & $1.368(3)$ \\
$\mathrm{F} 2-\mathrm{B} 1$ & $1.401(3)$ & $\mathrm{F} 4-\mathrm{B} 2$ & $1.380(3)$ \\
$\mathrm{O} 1-\mathrm{C} 10$ & $1.358(3)$ & $\mathrm{O} 2-\mathrm{C} 22$ & $1.382(3)$ \\
$\mathrm{N} 1-\mathrm{C} 1$ & $1.377(3)$ & $\mathrm{N} 4-\mathrm{C} 13$ & $1.380(3)$ \\
$\mathrm{N} 1-\mathrm{C} 2$ & $1.356(3)$ & $\mathrm{N} 4-\mathrm{C} 14$ & $1.353(3)$ \\
$\mathrm{N} 1-\mathrm{B} 1$ & $1.537(3)$ & $\mathrm{N} 4-\mathrm{B} 2$ & $1.545(3)$ \\
$\mathrm{N} 2-\mathrm{N} 3$ & $1.318(3)$ & $\mathrm{N} 5-\mathrm{N} 6$ & $1.312(3)$ \\
$\mathrm{N} 2-\mathrm{C} 1$ & $1.343(3)$ & $\mathrm{N} 5-\mathrm{C} 13$ & $1.338(3)$ \\
$\mathrm{N} 3-\mathrm{C} 7$ & $1.406(3)$ & $\mathrm{N} 6-\mathrm{C} 19$ & $1.416(3)$ \\
$\mathrm{N} 3-\mathrm{B} 1$ & $1.613(3)$ & $\mathrm{N} 6-\mathrm{B} 2$ & $1.618(3)$ \\
$\mathrm{C} 1-\mathrm{C} 4$ & $1.411(3)$ & $\mathrm{C} 13-\mathrm{C} 16$ & $1.415(3)$ \\
$\mathrm{C} 2-\mathrm{C} 3$ & $1.405(3)$ & $\mathrm{C} 14-\mathrm{C} 15$ & $1.408(3)$ \\
$\mathrm{C} 3-\mathrm{C} 4$ & $1.389(3)$ & $\mathrm{C} 15-\mathrm{C} 16$ & $1.389(3)$ \\
\hline
\end{tabular}

$\mathrm{C} 4, \mathrm{C} 2-\mathrm{C} 3, \mathrm{C} 13-\mathrm{C} 16$ and $\mathrm{C} 14-\mathrm{C} 15$ bonds are lengthened, while the $\mathrm{C} 3-\mathrm{C} 4$ and $\mathrm{C} 15-\mathrm{C} 16$ bonds are shortened compared to the normal bond lengths in pyrrole. This indicates that the azopyrrole moiety of the title compound must be in the hydrazone form (Chen et al., 2014).

\section{Supramolecular features and Hirshfeld analysis}

The two conformers also show supramolecular differences. One of the conformers only has a hydrogen bond between its hydroxyl group and that of the other conformer molecule (Fig. 1), whereas the hydroxyl group in the other conformer is also involved in intermolecular $\mathrm{O}-\mathrm{H} \cdots \mathrm{F}$ interactions (Fig. 2, Table 2), forming a one-dimensional ladder-like structure

Figure 2

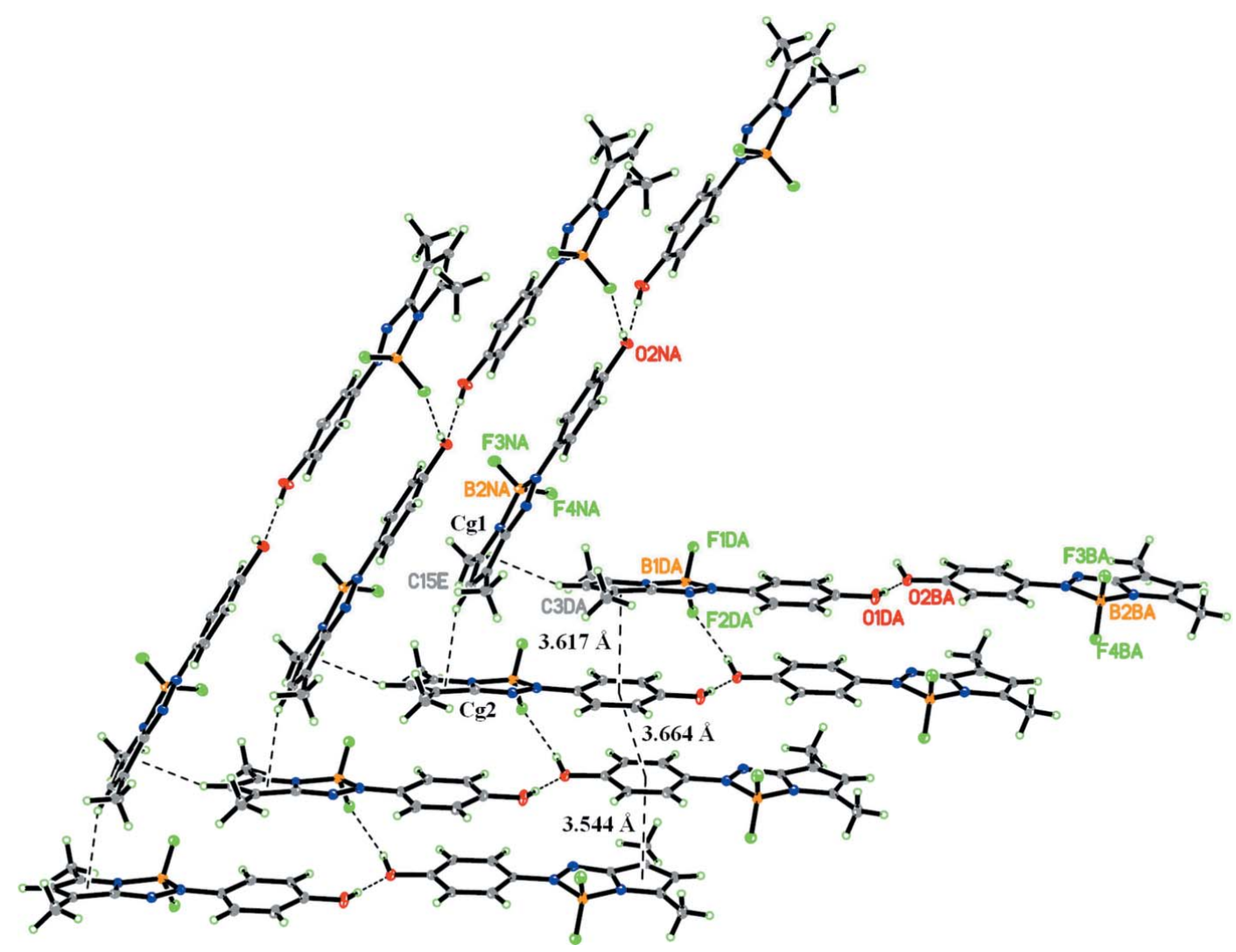

Part of the crystal packing showing molecules linked by $\mathrm{O}-\mathrm{H} \cdots \mathrm{O}$ and $\mathrm{O}-\mathrm{H} \cdots \mathrm{F}$ hydrogen bonds, $\pi-\pi$ interactions and $\mathrm{C}-\mathrm{H} \cdots \pi$ contacts. 
Table 2

Hydrogen-bond geometry $\left(\AA,^{\circ}\right)$.

$C g 2$ and $C g 6$ are the centroids of the N4/C13-C16 and N1/C1-C4 rings, respectively.

\begin{tabular}{lllll}
\hline$D-\mathrm{H} \cdots A$ & $D-\mathrm{H}$ & $\mathrm{H} \cdots A$ & $D \cdots A$ & $D-\mathrm{H} \cdots A$ \\
\hline $\mathrm{O} 1-\mathrm{H} 1 \cdots \mathrm{O} 2$ & 0.82 & 1.98 & $2.797(2)$ & 178 \\
$\mathrm{O} 2-\mathrm{H} 2 \cdots \mathrm{F} 2^{\mathrm{i}}$ & 0.82 & 2.06 & $2.812(2)$ & 152 \\
$\mathrm{C} 3-\mathrm{H} 3 \cdots C g 1^{\mathrm{ii}}$ & 0.93 & 2.62 & $3.501(2)$ & 158 \\
$\mathrm{C} 15-\mathrm{H} 15 \cdots C g 2^{\mathrm{iii}}$ & 0.93 & 2.63 & $3.506(2)$ & 157 \\
\hline
\end{tabular}

Symmetry codes: (i) $x-1, y, z$; (ii) $-x+\frac{3}{2}, y+\frac{1}{2},-z+\frac{3}{2}$; (iii) $-x+\frac{1}{2}, y-\frac{1}{2},-z+\frac{3}{2}$.

along [100]. In the ladder structure, the molecules are arranged in a parallel manner through $\pi-\pi$ interactions $[C g 1 \cdots C g 4(x-1, y, z)=3.544(1) \AA, C g 2 \cdots C g 3(1+x, y, z)=$ 3.617 (1) $\AA$ and $C g 3 \cdots C g 4(1+x, y, z)=3.664$ (13) $\AA$; $C g 1$, $C g 2, C g 3$ and $C g 4$ are the centroids of the N1/C1-C4, C7-C12 and $\mathrm{C} 19-\mathrm{C} 24$ rings, respectively]. The ladders assemble into a layer structure through $\mathrm{C}-\mathrm{H} \cdots \pi$ contacts (Table 2).
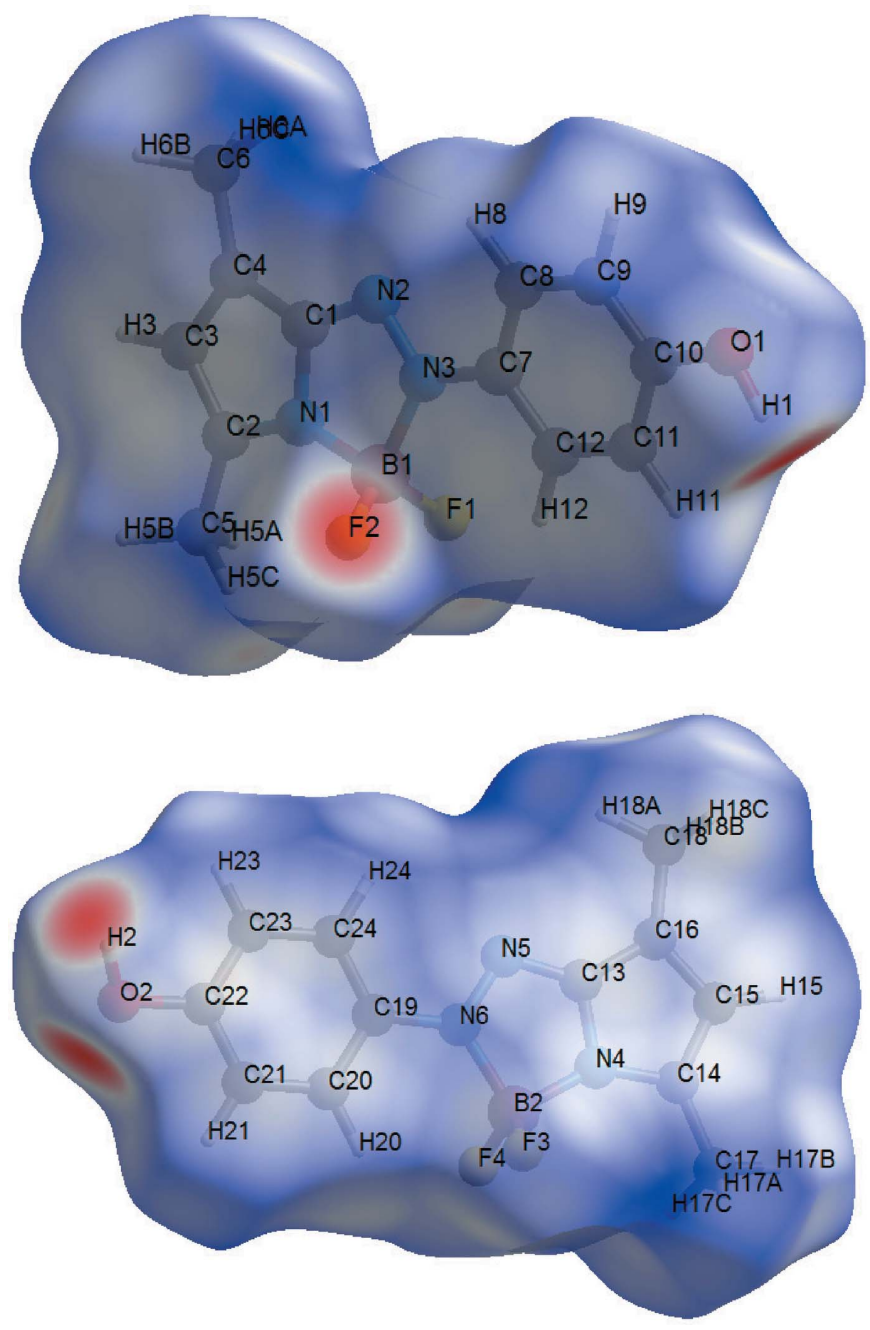

Figure 3

Hirshfeld surfaces of the two conformers mapped over $d_{\text {norm }}$ in the range -0.614 to 1.350 a.u. The intermolecular contacts can be seen in red regions.
Table 3

Experimental details.

Crystal data

Chemical formula

$M_{\mathrm{r}}$

Crystal system, space group

Temperature (K)

$a, b, c(\AA)$

$\beta\left({ }^{\circ}\right)$

$V\left(\AA^{3}\right)$

$Z$

Radiation type

$\mu\left(\mathrm{mm}^{-1}\right)$

Crystal size (mm)

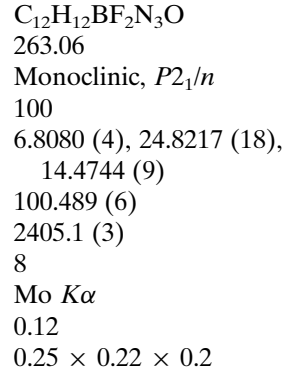

Data collection

Diffractometer

Absorption correction

$T_{\min }, T_{\max }$

No. of measured, independent and observed $[I>2 \sigma(I)]$ reflections

$R_{\text {int }}$

$(\sin \theta / \lambda)_{\max }\left(\AA^{-1}\right)$
Rigaku Oxford Diffraction Super- Nova, Dual, $\mathrm{Cu}$ at zero, Atlas S2 Multi-scan (CrysAlis PRO; Rigaku OD, 2015)
$0.680,1.000$
$11903,4228,3277$
0.043
0.595
4228
349
$\mathrm{H}$-atom parameters constrained $0.25,-0.26$

Refinement

$R\left[F^{2}>2 \sigma\left(F^{2}\right)\right], w R\left(F^{2}\right), S \quad 0.048,0.116,1.06$

No. of reflections

No. of parameters

$\mathrm{H}$-atom treatment

$\Delta \rho_{\max }, \Delta \rho_{\min }\left(\mathrm{e} \AA^{-3}\right)$

Computer programs: CrysAlis PRO (Rigaku OD, 2015), SHELXT (Sheldrick, 2015a), SHELXL2014 (Sheldrick, 2015b), OLEX2 (Dolomanov et al., 2009).

The Hirshfeld surfaces of the two conformers were generated using CrystalExplorer (Turner et al., 2017). Fig. 3 clearly shows that the two conformers are involved in different supramolecular interactions.

\section{Database survey}

A search in the Cambridge Structural Database (Version 5.38; Groom et al., 2016) for azopyrrole boron difluoride compounds returned two entries, 2,5-bis(4-dimethylaminophenylazo)pyrrole boron difluoride ( $\mathrm{Li}$ et al., 2009) and 2-(dimethylaminophenylazo)-5-ethyl-pyrrole boron difluoride (Lee et al., 2012). In both, the boron atoms have same coordination as in the title compound. The $\mathrm{N}-\mathrm{N}$ bonds also adopt trans conformations and their lengths [1.322(2) and 1.310 (1) $\AA$ ] are comparable to those in the title compound.

\section{Synthesis and crystallization}

To a solution of 2-(4-hydroxylphenylazo)-3,5-dimethyl-1- $\mathrm{H}$ pyrrole $(2 \mathrm{mmol}, 0.43 \mathrm{~g})$ and triethylamine $(6 \mathrm{~mL})$ in dry dichloromethane $(15 \mathrm{~mL})$ was slowly added boron trifluoride ethyl ether $(2 \mathrm{~mL})$. The resulting solution was stirred for $40 \mathrm{~min}$, and then saturated potassium carbonate solution was added and stirred for 30 minutes. The resulting solution was extracted with ethyl acetate $(10 \mathrm{~mL} \times 3)$ and evaporated under vacuum to dryness. The residue was purified by column chromatography, eluting with ethyl acetate and petroleum 
ether $(v / v=1: 14)$, to give a dark-green product, m.p. $=405 \mathrm{~K}$. Yield $65 \%$. ${ }^{1} \mathrm{H}$ NMR (400 MHz, DMSO- $\left.d_{6}\right): \delta 10.118(s, 1 \mathrm{H},-$ $\mathrm{OH}), 7.548-7.526(d, 2 \mathrm{H}, J=8.8 \mathrm{~Hz}, \mathrm{Ar}-\mathrm{CH}), 6.920-6.897(d$, $2 \mathrm{H}, J=9.2 \mathrm{~Hz}, \mathrm{Ar}-\mathrm{CH}), 6.342(s, 1 \mathrm{H}$, pyrrole-CH), 2.371( $s$, $\left.3 \mathrm{H},-\mathrm{CH}_{3}\right), 2.314\left(s, 3 \mathrm{H},-\mathrm{CH}_{3}\right)$. Suitable crystals for X-ray diffraction analysis were obtained by the slow evaporation of an $\mathrm{CHCl}_{3} / \mathrm{CH}_{3} \mathrm{OH}$ solution of the title compound.

\section{Refinement}

Crystal data, data collection and structure refinement details are summarized in Table 3. $\mathrm{OH} \mathrm{H}$ atoms were located from difference-Fourier maps and refined freely. Other $\mathrm{H}$ atoms were placed in calculated positions $(\mathrm{C}-\mathrm{H}=0.93$ or $0.96 \AA)$ and refined using a riding model, with $U_{\text {iso }}(\mathrm{H})=1.2 U_{\text {eq }}(\mathrm{C})$ or $1.5 U_{\text {eq }}(\mathrm{C}-$ methyl $)$.

\section{Funding information}

Funding for this research was provided by: National Natural Science Foundation of China (No. 21172174).

\section{References}

Chen, J. \& Yin, Z. (2014). Dyes Pigments, 102, 94-99.

Dolomanov, O. V., Bourhis, L. J., Gildea, R. J., Howard, J. A. K. \& Puschmann, H. (2009). J. Appl. Cryst. 42, 339-341.

Groom, C. R., Bruno, I. J., Lightfoot, M. P. \& Ward, S. C. (2016). Acta Cryst. B72, 171-179.

Lee, H. Y., Olasz, A., Chen, C.-H. \& Lee, D. (2012). Org. Lett. 14, 6286-6289.

Li, Y., Patrick, B. O. \& Dolphin, D. (2009). J. Org. Chem. 74, 52375243.

Li, B., Zhang, G., Sun, S. \& Yin, Z. (2011). Acta Cryst. E67, o247.

Mikroyannidis, J. A., Kabanakis, A. N. D. V., Tsagkournos, D. V. P., Balraju, P. \& Sharma, G. D. (2010). J. Mater. Chem. 20, 6464-6471.

Mikroyannidis, J. A., Roy, M. S. \& Sharma, G. D. (2010). J. Power Sources, 195, 5391-5398.

Rigaku OD (2015). CrysAlis PRO. Rigaku Oxford Diffraction, Yarnton, England.

Sheldrick, G. M. (2015a). Acta Cryst. A71, 3-8.

Sheldrick, G. M. (2015b). Acta Cryst. C71, 3-8.

Turner, M. J., McKinnon, J. J., Wolff, S. K., Grimwood, D. J., Spackman, P. R., Jayatilaka, D. \& Spackman, M. A. (2017). CrystalExplorer17. University of Western Australia.

Yin, Z., Wang, W., Guo, J., Wang, J., He, J. \& Cheng, J.-P. (2008). CrystEngComm, 10, 957-959. 


\section{supporting information}

Acta Cryst. (2018). E74, 743-746 [https://doi.org/10.1107/S2056989018006229]

\section{Crystal structure of difluorido\{2-[(4-hydroxyphenyl)diazenyl]-3,5-dimethyl- pyrrolido\}boron}

\section{Huixiao Feng and Zhenming Yin}

Computing details

Data collection: CrysAlis PRO (Rigaku OD, 2015); cell refinement: CrysAlis PRO (Rigaku OD, 2015); data reduction: CrysAlis PRO (Rigaku OD, 2015); program(s) used to solve structure: SHELXT (Sheldrick, 2015a); program(s) used to refine structure: SHELXL2014 (Sheldrick, 2015b); molecular graphics: OLEX2 (Dolomanov et al., 2009); software used to prepare material for publication: OLEX2 (Dolomanov et al., 2009).

Difluorido\{2-[(4-hydroxyphenyl) diazenyl- $\left.\kappa N^{2}\right]-3,5$-dimethylpyrrolido- $\left.\ \kappa N\right\}$ boron

\section{Crystal data}

$\mathrm{C}_{12} \mathrm{H}_{12} \mathrm{BF}_{2} \mathrm{~N}_{3} \mathrm{O}$

$M_{r}=263.06$

Monoclinic, $P 2{ }_{1} / n$

$a=6.8080(4) \AA$

$b=24.8217(18) \AA$

$c=14.4744(9) \AA$

$\beta=100.489(6)^{\circ}$

$V=2405.1(3) \AA^{3}$

$Z=8$

\section{Data collection}

Rigaku Oxford Diffraction SuperNova, Dual, $\mathrm{Cu}$ at zero, Atlas S2 diffractometer

Radiation source: micro-focus sealed X-ray tube, SuperNova (Mo) X-ray Source

Mirror monochromator

Detector resolution: 5.2740 pixels $\mathrm{mm}^{-1}$

$\omega$ scans

Absorption correction: multi-scan

(CrysAlis PRO; Rigaku OD, 2015)

\section{Refinement}

Refinement on $F^{2}$

Least-squares matrix: full

$R\left[F^{2}>2 \sigma\left(F^{2}\right)\right]=0.048$

$w R\left(F^{2}\right)=0.116$

$S=1.06$

4228 reflections

349 parameters
$F(000)=1088$

$D_{\mathrm{x}}=1.453 \mathrm{Mg} \mathrm{m}^{-3}$

Mo $K \alpha$ radiation, $\lambda=0.71073 \AA$

Cell parameters from 3432 reflections

$\theta=4.1-28.6^{\circ}$

$\mu=0.12 \mathrm{~mm}^{-1}$

$T=100 \mathrm{~K}$

Block, dark green

$0.25 \times 0.22 \times 0.2 \mathrm{~mm}$

$T_{\min }=0.680, T_{\max }=1.000$

11903 measured reflections

4228 independent reflections

3277 reflections with $I>2 \sigma(I)$

$R_{\text {int }}=0.043$

$\theta_{\max }=25.0^{\circ}, \theta_{\min }=3.2^{\circ}$

$h=-7 \rightarrow 8$

$k=-29 \rightarrow 29$

$l=-17 \rightarrow 15$

0 restraints

Primary atom site location: dual

Hydrogen site location: inferred from neighbouring sites

$\mathrm{H}$-atom parameters constrained

$w=1 /\left[\sigma^{2}\left(F_{\mathrm{o}}^{2}\right)+(0.0388 P)^{2}+1.6938 P\right]$

where $P=\left(F_{\mathrm{o}}{ }^{2}+2 F_{\mathrm{c}}{ }^{2}\right) / 3$ 
$(\Delta / \sigma)_{\max }<0.001$

$\Delta \rho_{\min }=-0.26$ e $\AA^{-3}$

$\Delta \rho_{\max }=0.25$ e $\AA^{-3}$

Special details

Geometry. All esds (except the esd in the dihedral angle between two 1.s. planes) are estimated using the full covariance matrix. The cell esds are taken into account individually in the estimation of esds in distances, angles and torsion angles; correlations between esds in cell parameters are only used when they are defined by crystal symmetry. An approximate (isotropic) treatment of cell esds is used for estimating esds involving l.s. planes.

Fractional atomic coordinates and isotropic or equivalent isotropic displacement parameters $\left(\AA^{2}\right)$

\begin{tabular}{|c|c|c|c|c|}
\hline & $x$ & $y$ & $z$ & $U_{\text {iso }} * / U_{\text {eq }}$ \\
\hline $\mathrm{F} 1$ & $1.31342(18)$ & $0.53429(5)$ & $0.89956(9)$ & $0.0210(3)$ \\
\hline $\mathrm{F} 2$ & $1.46735(18)$ & $0.45513(5)$ & $0.87853(8)$ & $0.0205(3)$ \\
\hline $\mathrm{O} 1$ & $0.5707(2)$ & $0.40021(7)$ & $0.61520(11)$ & $0.0272(4)$ \\
\hline H1 & 0.5225 & 0.3893 & 0.6596 & $0.041^{*}$ \\
\hline N1 & $1.5952(3)$ & $0.53806(7)$ & $0.81633(12)$ & $0.0156(4)$ \\
\hline $\mathrm{N} 2$ & $1.3878(3)$ & $0.52607(8)$ & $0.67448(13)$ & 0.0178 (4) \\
\hline $\mathrm{N} 3$ & 1.2967 (3) & $0.50217(7)$ & $0.73622(13)$ & $0.0162(4)$ \\
\hline $\mathrm{C} 1$ & $1.5603(3)$ & $0.54666(9)$ & $0.72074(15)$ & $0.0162(5)$ \\
\hline $\mathrm{C} 2$ & $1.7710(3)$ & $0.56211(9)$ & $0.85288(16)$ & $0.0166(5)$ \\
\hline $\mathrm{C} 3$ & $1.8484(3)$ & $0.58566(9)$ & $0.77884(16)$ & $0.0182(5)$ \\
\hline $\mathrm{H} 3$ & 1.9686 & 0.6043 & 0.7852 & $0.022 *$ \\
\hline $\mathrm{C} 4$ & $1.7176(3)$ & $0.57669(9)$ & $0.69470(16)$ & $0.0180(5)$ \\
\hline $\mathrm{C} 5$ & $1.8540(3)$ & $0.56219(10)$ & $0.95504(15)$ & $0.0211(5)$ \\
\hline $\mathrm{H} 5 \mathrm{~A}$ & 1.8228 & 0.5958 & 0.9820 & $0.032 *$ \\
\hline H5B & 1.9964 & 0.5578 & 0.9645 & $0.032 *$ \\
\hline $\mathrm{H} 5 \mathrm{C}$ & 1.7965 & 0.5331 & 0.9848 & $0.032 *$ \\
\hline C6 & $1.7354(4)$ & $0.59469(11)$ & $0.59812(16)$ & $0.0257(6)$ \\
\hline H6A & 1.6381 & 0.5762 & 0.5528 & $0.038^{*}$ \\
\hline H6B & 1.8669 & 0.5866 & 0.5869 & $0.038^{*}$ \\
\hline $\mathrm{H} 6 \mathrm{C}$ & 1.7125 & 0.6328 & 0.5926 & $0.038^{*}$ \\
\hline $\mathrm{C} 7$ & $1.1152(3)$ & $0.47520(9)$ & $0.70490(15)$ & $0.0163(5)$ \\
\hline $\mathrm{C} 8$ & 1.0407 (3) & $0.46718(9)$ & $0.60966(16)$ & $0.0198(5)$ \\
\hline $\mathrm{H} 8$ & 1.1142 & 0.4786 & 0.5650 & $0.024 *$ \\
\hline C9 & $0.8590(3)$ & $0.44247(10)$ & $0.58158(16)$ & $0.0223(5)$ \\
\hline H9 & 0.8086 & 0.4380 & 0.5179 & $0.027^{*}$ \\
\hline $\mathrm{C} 10$ & $0.7495(3)$ & $0.42404(9)$ & 0.64779 (16) & $0.0190(5)$ \\
\hline C11 & $0.8254(3)$ & $0.43105(9)$ & $0.74289(16)$ & $0.0179(5)$ \\
\hline H11 & 0.7541 & 0.4185 & 0.7876 & $0.021^{*}$ \\
\hline $\mathrm{C} 12$ & $1.0063(3)$ & $0.45662(9)$ & $0.77097(15)$ & $0.0170(5)$ \\
\hline H12 & 1.0560 & 0.4615 & 0.8346 & $0.020^{*}$ \\
\hline B1 & $1.4183(4)$ & 0.50677 (11) & $0.84252(18)$ & $0.0174(6)$ \\
\hline F3 & $-0.50768(19)$ & $0.27493(6)$ & $0.54470(9)$ & $0.0247(3)$ \\
\hline $\mathrm{F} 4$ & $-0.33107(18)$ & $0.19699(6)$ & $0.55925(9)$ & $0.0237(3)$ \\
\hline $\mathrm{O} 2$ & $0.3980(2)$ & $0.36293(7)$ & $0.76431(11)$ & $0.0226(4)$ \\
\hline $\mathrm{H} 2$ & 0.4090 & 0.3820 & 0.8112 & $0.034 *$ \\
\hline N4 & $-0.6118(3)$ & $0.20404(8)$ & 0.64279 (13) & $0.0172(4)$ \\
\hline N5 & $-0.4053(3)$ & $0.23598(8)$ & $0.77401(13)$ & $0.0176(4)$ \\
\hline
\end{tabular}




$\begin{array}{lllll}\text { N6 } & -0.3216(3) & 0.25089(8) & 0.70328(12) & 0.0167(4) \\ \text { C13 } & -0.5729(3) & 0.20907(9) & 0.73934(15) & 0.0168(5) \\ \text { C14 } & -0.7850(3) & 0.17649(9) & 0.61832(16) & 0.0188(5) \\ \text { C15 } & -0.8566(3) & 0.16381(9) & 0.70106(16) & 0.0198(5) \\ \text { H15 } & -0.9737 & 0.1451 & 0.7036 & 0.024^{*} \\ \text { C16 } & -0.7251(3) & 0.18365(9) & 0.77825(16) & 0.0193(5) \\ \text { C17 } & -0.8717(3) & 0.16370(11) & 0.51897(17) & 0.0261(6) \\ \text { H17A } & -0.9208 & 0.1961 & 0.4867 & 0.039^{*} \\ \text { H17B } & -0.9797 & 0.1386 & 0.5171 & 0.039^{*} \\ \text { H17C } & -0.7707 & 0.1481 & 0.4889 & 0.039^{*} \\ \text { C18 } & -0.7344(4) & 0.17973(10) & 0.88049(16) & 0.0254(6) \\ \text { H18A } & -0.6142 & 0.1943 & 0.9169 & 0.038^{*} \\ \text { H18B } & -0.7476 & 0.1426 & 0.8971 & 0.038^{*} \\ \text { H18C } & -0.8474 & 0.1997 & 0.8931 & 0.038^{*} \\ \text { C19 } & -0.1401(3) & 0.28017(9) & 0.72225(15) & 0.0155(5) \\ \text { C20 } & -0.0500(3) & 0.29409(9) & 0.64649(16) & 0.0186(5) \\ \text { H20 } & -0.1105 & 0.2848 & 0.5857 & 0.022^{*} \\ \text { C21 } & 0.1291(3) & 0.32173(9) & 0.66166(16) & 0.0182(5) \\ \text { H21 } & 0.1898 & 0.3308 & 0.6112 & 0.022^{*} \\ \text { C22 } & 0.2179(3) & 0.33579(9) & 0.75205(16) & 0.0170(5) \\ \text { C23 } & 0.1294(3) & 0.32146(9) & 0.82758(16) & 0.0183(5) \\ \text { H23 } & 0.1907 & 0.3306 & 0.8883 & 0.022^{*} \\ \text { C24 } & -0.0496(3) & 0.29372(9) & 0.81286(15) & 0.0180(5) \\ \text { H24 } & -0.1090 & 0.2842 & 0.8635 & 0.022^{*} \\ \text { B2 } & -0.4440(4) & 0.23212(11) & 0.60191(18) & 0.0192(6) \\ & & & & \end{array}$

Atomic displacement parameters $\left(\AA^{2}\right)$

\begin{tabular}{lllllll}
\hline & $U^{11}$ & $U^{22}$ & $U^{33}$ & $U^{12}$ & $U^{13}$ & $U^{23}$ \\
\hline F1 & $0.0209(7)$ & $0.0223(8)$ & $0.0214(7)$ & $-0.0015(6)$ & $0.0079(5)$ & $-0.0030(6)$ \\
F2 & $0.0227(7)$ & $0.0171(7)$ & $0.0211(7)$ & $-0.0029(6)$ & $0.0027(5)$ & $0.0025(6)$ \\
O1 & $0.0236(9)$ & $0.0309(11)$ & $0.0260(9)$ & $-0.0135(8)$ & $0.0014(7)$ & $0.0018(8)$ \\
N1 & $0.0156(9)$ & $0.0132(10)$ & $0.0185(10)$ & $0.0009(8)$ & $0.0048(7)$ & $-0.0001(8)$ \\
N2 & $0.0182(10)$ & $0.0144(10)$ & $0.0221(10)$ & $-0.0002(8)$ & $0.0070(8)$ & $0.0008(8)$ \\
N3 & $0.0148(9)$ & $0.0148(10)$ & $0.0195(10)$ & $-0.0007(8)$ & $0.0047(8)$ & $0.0007(8)$ \\
C1 & $0.0174(11)$ & $0.0136(12)$ & $0.0176(12)$ & $0.0017(10)$ & $0.0031(9)$ & $0.0001(10)$ \\
C2 & $0.0141(11)$ & $0.0117(12)$ & $0.0243(12)$ & $0.0026(9)$ & $0.0040(9)$ & $-0.0012(10)$ \\
C3 & $0.0148(11)$ & $0.0128(12)$ & $0.0277(13)$ & $-0.0022(9)$ & $0.0058(9)$ & $-0.0003(10)$ \\
C4 & $0.0182(12)$ & $0.0129(12)$ & $0.0248(12)$ & $0.0002(10)$ & $0.0087(9)$ & $0.0005(10)$ \\
C5 & $0.0204(12)$ & $0.0209(13)$ & $0.0218(12)$ & $-0.0014(10)$ & $0.0037(9)$ & $0.0011(10)$ \\
C6 & $0.0244(13)$ & $0.0275(15)$ & $0.0262(13)$ & $-0.0037(11)$ & $0.0073(10)$ & $0.0049(11)$ \\
C7 & $0.0163(11)$ & $0.0117(12)$ & $0.0207(12)$ & $-0.0006(9)$ & $0.0033(9)$ & $0.0013(10)$ \\
C8 & $0.0220(12)$ & $0.0185(13)$ & $0.0198(12)$ & $-0.0030(10)$ & $0.0061(9)$ & $0.0021(10)$ \\
C9 & $0.0265(13)$ & $0.0220(14)$ & $0.0169(12)$ & $-0.0048(11)$ & $0.0002(9)$ & $-0.0001(10)$ \\
C10 & $0.0146(11)$ & $0.0152(13)$ & $0.0263(13)$ & $-0.0016(10)$ & $0.0009(9)$ & $0.0007(10)$ \\
C11 & $0.0161(11)$ & $0.0167(12)$ & $0.0219(12)$ & $0.0019(10)$ & $0.0066(9)$ & $0.0032(10)$ \\
C12 & $0.0187(12)$ & $0.0155(12)$ & $0.0168(11)$ & $-0.0004(10)$ & $0.0032(9)$ & $-0.0006(10)$ \\
B1 & $0.0169(13)$ & $0.0168(14)$ & $0.0185(13)$ & $-0.0009(11)$ & $0.0031(10)$ & $0.0016(11)$
\end{tabular}




\begin{tabular}{lllllll} 
F3 & $0.0265(7)$ & $0.0242(8)$ & $0.0230(7)$ & $-0.0026(6)$ & $0.0035(6)$ & $0.0065(6)$ \\
F4 & $0.0227(7)$ & $0.0267(8)$ & $0.0234(7)$ & $-0.0033(6)$ & $0.0089(6)$ & $-0.0067(6)$ \\
O2 & $0.0197(8)$ & $0.0208(10)$ & $0.0278(9)$ & $-0.0056(7)$ & $0.0056(7)$ & $-0.0043(8)$ \\
N4 & $0.0170(10)$ & $0.0160(11)$ & $0.0190(10)$ & $-0.0010(8)$ & $0.0049(8)$ & $0.0002(8)$ \\
N5 & $0.0191(10)$ & $0.0141(10)$ & $0.0211(10)$ & $0.0006(8)$ & $0.0073(8)$ & $-0.0002(8)$ \\
N6 & $0.0181(10)$ & $0.0139(10)$ & $0.0193(10)$ & $-0.0016(8)$ & $0.0064(8)$ & $0.0009(8)$ \\
C13 & $0.0171(12)$ & $0.0132(12)$ & $0.0210(12)$ & $0.0021(10)$ & $0.0063(9)$ & $0.0006(10)$ \\
C14 & $0.0159(11)$ & $0.0142(12)$ & $0.0266(13)$ & $0.0009(10)$ & $0.0047(9)$ & $0.0010(10)$ \\
C15 & $0.0143(11)$ & $0.0153(12)$ & $0.0309(13)$ & $-0.0009(10)$ & $0.0065(10)$ & $0.0020(11)$ \\
C16 & $0.0208(12)$ & $0.0137(12)$ & $0.0250(12)$ & $0.0029(10)$ & $0.0084(10)$ & $0.0030(10)$ \\
C17 & $0.0200(12)$ & $0.0292(15)$ & $0.0280(14)$ & $-0.0039(11)$ & $0.0018(10)$ & $0.0011(12)$ \\
C18 & $0.0283(13)$ & $0.0231(14)$ & $0.0275(13)$ & $0.0005(11)$ & $0.0123(10)$ & $0.0035(11)$ \\
C19 & $0.0148(11)$ & $0.0101(12)$ & $0.0220(12)$ & $-0.0003(9)$ & $0.0046(9)$ & $0.0000(10)$ \\
C20 & $0.0225(12)$ & $0.0171(13)$ & $0.0162(11)$ & $-0.0015(10)$ & $0.0039(9)$ & $-0.0007(10)$ \\
C21 & $0.0197(12)$ & $0.0147(12)$ & $0.0223(12)$ & $0.0007(10)$ & $0.0094(9)$ & $0.0025(10)$ \\
C22 & $0.0145(11)$ & $0.0130(12)$ & $0.0237(12)$ & $0.0001(9)$ & $0.0039(9)$ & $-0.0003(10)$ \\
C23 & $0.0196(12)$ & $0.0146(12)$ & $0.0191(12)$ & $0.0001(10)$ & $-0.0004(9)$ & $-0.0028(10)$ \\
C24 & $0.0194(12)$ & $0.0169(13)$ & $0.0193(12)$ & $0.0005(10)$ & $0.0079(9)$ & $0.0018(10)$ \\
B2 & $0.0203(13)$ & $0.0203(15)$ & $0.0178(13)$ & $-0.0031(12)$ & $0.0059(10)$ & $0.0004(12)$ \\
& & & & & & \\
\hline
\end{tabular}

Geometric parameters $\left(\AA,{ }^{\circ}\right)$

\begin{tabular}{llll}
\hline $\mathrm{F} 1-\mathrm{B} 1$ & $1.369(3)$ & $\mathrm{F} 3-\mathrm{B} 2$ & $1.368(3)$ \\
$\mathrm{F} 2-\mathrm{B} 1$ & $1.401(3)$ & $\mathrm{F} 4-\mathrm{B} 2$ & $1.380(3)$ \\
$\mathrm{O} 1-\mathrm{H} 1$ & 0.8200 & $\mathrm{O} 2-\mathrm{H} 2$ & 0.8200 \\
$\mathrm{O} 1-\mathrm{C} 10$ & $1.358(3)$ & $\mathrm{O} 2-\mathrm{C} 22$ & $1.382(3)$ \\
$\mathrm{N} 1-\mathrm{C} 1$ & $1.377(3)$ & $\mathrm{N} 4-\mathrm{C} 13$ & $1.380(3)$ \\
$\mathrm{N} 1-\mathrm{C} 2$ & $1.356(3)$ & $\mathrm{N} 4-\mathrm{C} 14$ & $1.353(3)$ \\
$\mathrm{N} 1-\mathrm{B} 1$ & $1.537(3)$ & $\mathrm{N} 4-\mathrm{B} 2$ & $1.545(3)$ \\
$\mathrm{N} 2-\mathrm{N} 3$ & $1.318(3)$ & $\mathrm{N} 5-\mathrm{N} 6$ & $1.312(3)$ \\
$\mathrm{N} 2-\mathrm{C} 1$ & $1.343(3)$ & $\mathrm{N} 5-\mathrm{C} 13$ & $1.338(3)$ \\
$\mathrm{N} 3-\mathrm{C} 7$ & $1.406(3)$ & $\mathrm{N} 6-\mathrm{C} 19$ & $1.416(3)$ \\
$\mathrm{N} 3-\mathrm{B} 1$ & $1.613(3)$ & $\mathrm{N} 6-\mathrm{B} 2$ & $1.618(3)$ \\
$\mathrm{C} 1-\mathrm{C} 4$ & $1.411(3)$ & $\mathrm{C} 13-\mathrm{C} 16$ & $1.415(3)$ \\
$\mathrm{C} 2-\mathrm{C} 3$ & $1.405(3)$ & $\mathrm{C} 14-\mathrm{C} 15$ & $1.408(3)$ \\
$\mathrm{C} 2-\mathrm{C} 5$ & $1.484(3)$ & $\mathrm{C} 14-\mathrm{C} 17$ & $1.486(3)$ \\
$\mathrm{C} 3-\mathrm{H} 3$ & 0.9300 & $\mathrm{C} 15-\mathrm{H} 15$ & 0.9300 \\
$\mathrm{C} 3-\mathrm{C} 4$ & $1.389(3)$ & $\mathrm{C} 15-\mathrm{C} 16$ & $1.389(3)$ \\
$\mathrm{C} 4-\mathrm{C} 6$ & $1.493(3)$ & $\mathrm{C} 16-\mathrm{C} 18$ & $1.496(3)$ \\
$\mathrm{C} 5-\mathrm{H} 5 \mathrm{~A}$ & 0.9600 & $\mathrm{C} 17-\mathrm{H} 17 \mathrm{~A}$ & 0.9600 \\
$\mathrm{C} 5-\mathrm{H} 5 \mathrm{~B}$ & 0.9600 & $\mathrm{C} 17-\mathrm{H} 17 \mathrm{~B}$ & 0.9600 \\
$\mathrm{C} 5-\mathrm{H} 5 \mathrm{C}$ & 0.9600 & $\mathrm{C} 17-\mathrm{H} 17 \mathrm{C}$ & 0.9600 \\
$\mathrm{C} 6-\mathrm{H} 6 \mathrm{~A}$ & 0.9600 & $\mathrm{C} 18-\mathrm{H} 18 \mathrm{~A}$ & 0.9600 \\
$\mathrm{C} 6-\mathrm{H} 6 \mathrm{~B}$ & 0.9600 & $\mathrm{C} 18-\mathrm{H} 18 \mathrm{~B}$ & 0.9600 \\
$\mathrm{C} 6-\mathrm{H} 6 \mathrm{C}$ & 0.9600 & $\mathrm{C} 18-\mathrm{H} 18 \mathrm{C}$ & 0.9600 \\
$\mathrm{C} 7-\mathrm{C} 8$ & $1.394(3)$ & $\mathrm{C} 19-\mathrm{C} 20$ & $1.394(3)$ \\
$\mathrm{C} 7-\mathrm{C} 12$ & $1.391(3)$ & $\mathrm{C} 19-\mathrm{C} 24$ & $1.385(3)$ \\
$\mathrm{C} 8-\mathrm{H} 8$ & 0.9300 & $\mathrm{C} 20-\mathrm{H} 20$ & 0.9300
\end{tabular}




\begin{tabular}{|c|c|c|c|}
\hline $\mathrm{C} 8-\mathrm{C} 9$ & $1.375(3)$ & $\mathrm{C} 20-\mathrm{C} 21$ & $1.382(3)$ \\
\hline C9-H9 & 0.9300 & $\mathrm{C} 21-\mathrm{H} 21$ & 0.9300 \\
\hline $\mathrm{C} 9-\mathrm{C} 10$ & $1.394(3)$ & $\mathrm{C} 21-\mathrm{C} 22$ & $1.382(3)$ \\
\hline $\mathrm{C} 10-\mathrm{C} 11$ & $1.390(3)$ & $\mathrm{C} 22-\mathrm{C} 23$ & $1.387(3)$ \\
\hline $\mathrm{C} 11-\mathrm{H} 11$ & 0.9300 & $\mathrm{C} 23-\mathrm{H} 23$ & 0.9300 \\
\hline $\mathrm{C} 11-\mathrm{C} 12$ & $1.380(3)$ & $\mathrm{C} 23-\mathrm{C} 24$ & $1.382(3)$ \\
\hline $\mathrm{C} 12-\mathrm{H} 12$ & 0.9300 & $\mathrm{C} 24-\mathrm{H} 24$ & 0.9300 \\
\hline $\mathrm{C} 10-\mathrm{O} 1-\mathrm{H} 1$ & 109.5 & $\mathrm{C} 22-\mathrm{O} 2-\mathrm{H} 2$ & 109.5 \\
\hline $\mathrm{C} 1-\mathrm{N} 1-\mathrm{B} 1$ & $109.05(17)$ & $\mathrm{C} 13-\mathrm{N} 4-\mathrm{B} 2$ & $109.16(18)$ \\
\hline $\mathrm{C} 2-\mathrm{N} 1-\mathrm{C} 1$ & $107.60(18)$ & $\mathrm{C} 14-\mathrm{N} 4-\mathrm{C} 13$ & $107.95(18)$ \\
\hline $\mathrm{C} 2-\mathrm{N} 1-\mathrm{B} 1$ & $143.30(19)$ & $\mathrm{C} 14-\mathrm{N} 4-\mathrm{B} 2$ & $142.88(19)$ \\
\hline $\mathrm{N} 3-\mathrm{N} 2-\mathrm{C} 1$ & $108.06(18)$ & $\mathrm{N} 6-\mathrm{N} 5-\mathrm{C} 13$ & $108.05(18)$ \\
\hline $\mathrm{N} 2-\mathrm{N} 3-\mathrm{C} 7$ & $119.34(18)$ & $\mathrm{N} 5-\mathrm{N} 6-\mathrm{C} 19$ & $118.74(18)$ \\
\hline $\mathrm{N} 2-\mathrm{N} 3-\mathrm{B} 1$ & $113.07(17)$ & $\mathrm{N} 5-\mathrm{N} 6-\mathrm{B} 2$ & $113.64(17)$ \\
\hline $\mathrm{C} 7-\mathrm{N} 3-\mathrm{B} 1$ & $127.56(18)$ & $\mathrm{C} 19-\mathrm{N} 6-\mathrm{B} 2$ & $127.62(18)$ \\
\hline $\mathrm{N} 1-\mathrm{C} 1-\mathrm{C} 4$ & $110.47(18)$ & $\mathrm{N} 4-\mathrm{C} 13-\mathrm{C} 16$ & $110.09(19)$ \\
\hline $\mathrm{N} 2-\mathrm{C} 1-\mathrm{N} 1$ & $114.66(19)$ & $\mathrm{N} 5-\mathrm{C} 13-\mathrm{N} 4$ & $114.72(19)$ \\
\hline $\mathrm{N} 2-\mathrm{C} 1-\mathrm{C} 4$ & $134.9(2)$ & $\mathrm{N} 5-\mathrm{C} 13-\mathrm{C} 16$ & $135.2(2)$ \\
\hline $\mathrm{N} 1-\mathrm{C} 2-\mathrm{C} 3$ & $108.23(19)$ & $\mathrm{N} 4-\mathrm{C} 14-\mathrm{C} 15$ & $108.08(19)$ \\
\hline $\mathrm{N} 1-\mathrm{C} 2-\mathrm{C} 5$ & $122.6(2)$ & $\mathrm{N} 4-\mathrm{C} 14-\mathrm{C} 17$ & $122.4(2)$ \\
\hline $\mathrm{C} 3-\mathrm{C} 2-\mathrm{C} 5$ & $129.2(2)$ & $\mathrm{C} 15-\mathrm{C} 14-\mathrm{C} 17$ & $129.6(2)$ \\
\hline $\mathrm{C} 2-\mathrm{C} 3-\mathrm{H} 3$ & 125.3 & $\mathrm{C} 14-\mathrm{C} 15-\mathrm{H} 15$ & 125.3 \\
\hline $\mathrm{C} 4-\mathrm{C} 3-\mathrm{C} 2$ & $109.40(19)$ & $\mathrm{C} 16-\mathrm{C} 15-\mathrm{C} 14$ & $109.4(2)$ \\
\hline $\mathrm{C} 4-\mathrm{C} 3-\mathrm{H} 3$ & 125.3 & $\mathrm{C} 16-\mathrm{C} 15-\mathrm{H} 15$ & 125.3 \\
\hline $\mathrm{C} 1-\mathrm{C} 4-\mathrm{C} 6$ & $127.1(2)$ & $\mathrm{C} 13-\mathrm{C} 16-\mathrm{C} 18$ & $126.1(2)$ \\
\hline $\mathrm{C} 3-\mathrm{C} 4-\mathrm{C} 1$ & $104.30(19)$ & $\mathrm{C} 15-\mathrm{C} 16-\mathrm{C} 13$ & $104.4(2)$ \\
\hline $\mathrm{C} 3-\mathrm{C} 4-\mathrm{C} 6$ & $128.6(2)$ & $\mathrm{C} 15-\mathrm{C} 16-\mathrm{C} 18$ & $129.5(2)$ \\
\hline $\mathrm{C} 2-\mathrm{C} 5-\mathrm{H} 5 \mathrm{~A}$ & 109.5 & $\mathrm{C} 14-\mathrm{C} 17-\mathrm{H} 17 \mathrm{~A}$ & 109.5 \\
\hline $\mathrm{C} 2-\mathrm{C} 5-\mathrm{H} 5 \mathrm{~B}$ & 109.5 & $\mathrm{C} 14-\mathrm{C} 17-\mathrm{H} 17 \mathrm{~B}$ & 109.5 \\
\hline $\mathrm{C} 2-\mathrm{C} 5-\mathrm{H} 5 \mathrm{C}$ & 109.5 & $\mathrm{C} 14-\mathrm{C} 17-\mathrm{H} 17 \mathrm{C}$ & 109.5 \\
\hline $\mathrm{H} 5 \mathrm{~A}-\mathrm{C} 5-\mathrm{H} 5 \mathrm{~B}$ & 109.5 & $\mathrm{H} 17 \mathrm{~A}-\mathrm{C} 17-\mathrm{H} 17 \mathrm{~B}$ & 109.5 \\
\hline $\mathrm{H} 5 \mathrm{~A}-\mathrm{C} 5-\mathrm{H} 5 \mathrm{C}$ & 109.5 & $\mathrm{H} 17 \mathrm{~A}-\mathrm{C} 17-\mathrm{H} 17 \mathrm{C}$ & 109.5 \\
\hline $\mathrm{H} 5 \mathrm{~B}-\mathrm{C} 5-\mathrm{H} 5 \mathrm{C}$ & 109.5 & $\mathrm{H} 17 \mathrm{~B}-\mathrm{C} 17-\mathrm{H} 17 \mathrm{C}$ & 109.5 \\
\hline $\mathrm{C} 4-\mathrm{C} 6-\mathrm{H} 6 \mathrm{~A}$ & 109.5 & $\mathrm{C} 16-\mathrm{C} 18-\mathrm{H} 18 \mathrm{~A}$ & 109.5 \\
\hline $\mathrm{C} 4-\mathrm{C} 6-\mathrm{H} 6 \mathrm{~B}$ & 109.5 & $\mathrm{C} 16-\mathrm{C} 18-\mathrm{H} 18 \mathrm{~B}$ & 109.5 \\
\hline $\mathrm{C} 4-\mathrm{C} 6-\mathrm{H} 6 \mathrm{C}$ & 109.5 & $\mathrm{C} 16-\mathrm{C} 18-\mathrm{H} 18 \mathrm{C}$ & 109.5 \\
\hline $\mathrm{H} 6 \mathrm{~A}-\mathrm{C} 6-\mathrm{H} 6 \mathrm{~B}$ & 109.5 & $\mathrm{H} 18 \mathrm{~A}-\mathrm{C} 18-\mathrm{H} 18 \mathrm{~B}$ & 109.5 \\
\hline $\mathrm{H} 6 \mathrm{~A}-\mathrm{C} 6-\mathrm{H} 6 \mathrm{C}$ & 109.5 & $\mathrm{H} 18 \mathrm{~A}-\mathrm{C} 18-\mathrm{H} 18 \mathrm{C}$ & 109.5 \\
\hline $\mathrm{H} 6 \mathrm{~B}-\mathrm{C} 6-\mathrm{H} 6 \mathrm{C}$ & 109.5 & $\mathrm{H} 18 \mathrm{~B}-\mathrm{C} 18-\mathrm{H} 18 \mathrm{C}$ & 109.5 \\
\hline $\mathrm{C} 8-\mathrm{C} 7-\mathrm{N} 3$ & $121.8(2)$ & $\mathrm{C} 20-\mathrm{C} 19-\mathrm{N} 6$ & $117.90(19)$ \\
\hline $\mathrm{C} 12-\mathrm{C} 7-\mathrm{N} 3$ & $118.89(19)$ & $\mathrm{C} 24-\mathrm{C} 19-\mathrm{N} 6$ & $122.0(2)$ \\
\hline $\mathrm{C} 12-\mathrm{C} 7-\mathrm{C} 8$ & $119.3(2)$ & $\mathrm{C} 24-\mathrm{C} 19-\mathrm{C} 20$ & $120.1(2)$ \\
\hline $\mathrm{C} 7-\mathrm{C} 8-\mathrm{H} 8$ & 119.9 & $\mathrm{C} 19-\mathrm{C} 20-\mathrm{H} 20$ & 120.0 \\
\hline $\mathrm{C} 9-\mathrm{C} 8-\mathrm{C} 7$ & $120.1(2)$ & $\mathrm{C} 21-\mathrm{C} 20-\mathrm{C} 19$ & $120.0(2)$ \\
\hline $\mathrm{C} 9-\mathrm{C} 8-\mathrm{H} 8$ & 119.9 & $\mathrm{C} 21-\mathrm{C} 20-\mathrm{H} 20$ & 120.0 \\
\hline $\mathrm{C} 8-\mathrm{C} 9-\mathrm{H} 9$ & 119.7 & $\mathrm{C} 20-\mathrm{C} 21-\mathrm{H} 21$ & 120.1 \\
\hline $\mathrm{C} 8-\mathrm{C} 9-\mathrm{C} 10$ & $120.6(2)$ & $\mathrm{C} 20-\mathrm{C} 21-\mathrm{C} 22$ & $119.8(2)$ \\
\hline
\end{tabular}




\begin{tabular}{|c|c|c|c|}
\hline $\mathrm{C} 10-\mathrm{C} 9-\mathrm{H} 9$ & 119.7 & $\mathrm{C} 22-\mathrm{C} 21-\mathrm{H} 21$ & 120.1 \\
\hline $\mathrm{O} 1-\mathrm{C} 10-\mathrm{C} 9$ & $117.5(2)$ & $\mathrm{O} 2-\mathrm{C} 22-\mathrm{C} 21$ & $118.0(2)$ \\
\hline $\mathrm{O} 1-\mathrm{C} 10-\mathrm{C} 11$ & $123.1(2)$ & $\mathrm{O} 2-\mathrm{C} 22-\mathrm{C} 23$ & $121.8(2)$ \\
\hline $\mathrm{C} 11-\mathrm{C} 10-\mathrm{C} 9$ & $119.4(2)$ & $\mathrm{C} 21-\mathrm{C} 22-\mathrm{C} 23$ & $120.2(2)$ \\
\hline $\mathrm{C} 10-\mathrm{C} 11-\mathrm{H} 11$ & 120.0 & $\mathrm{C} 22-\mathrm{C} 23-\mathrm{H} 23$ & 119.9 \\
\hline $\mathrm{C} 12-\mathrm{C} 11-\mathrm{C} 10$ & $120.0(2)$ & $\mathrm{C} 24-\mathrm{C} 23-\mathrm{C} 22$ & $120.2(2)$ \\
\hline $\mathrm{C} 12-\mathrm{C} 11-\mathrm{H} 11$ & 120.0 & $\mathrm{C} 24-\mathrm{C} 23-\mathrm{H} 23$ & 119.9 \\
\hline $\mathrm{C} 7-\mathrm{C} 12-\mathrm{H} 12$ & 119.7 & $\mathrm{C} 19-\mathrm{C} 24-\mathrm{H} 24$ & 120.2 \\
\hline $\mathrm{C} 11-\mathrm{C} 12-\mathrm{C} 7$ & $120.6(2)$ & $\mathrm{C} 23-\mathrm{C} 24-\mathrm{C} 19$ & $119.7(2)$ \\
\hline $\mathrm{C} 11-\mathrm{C} 12-\mathrm{H} 12$ & 119.7 & $\mathrm{C} 23-\mathrm{C} 24-\mathrm{H} 24$ & 120.2 \\
\hline $\mathrm{F} 1-\mathrm{B} 1-\mathrm{F} 2$ & $110.33(19)$ & $\mathrm{F} 3-\mathrm{B} 2-\mathrm{F} 4$ & $111.15(19)$ \\
\hline $\mathrm{F} 1-\mathrm{B} 1-\mathrm{N} 1$ & $114.5(2)$ & $\mathrm{F} 3-\mathrm{B} 2-\mathrm{N} 4$ & $114.04(19)$ \\
\hline $\mathrm{F} 1-\mathrm{B} 1-\mathrm{N} 3$ & $112.16(18)$ & $\mathrm{F} 3-\mathrm{B} 2-\mathrm{N} 6$ & $112.3(2)$ \\
\hline $\mathrm{F} 2-\mathrm{B} 1-\mathrm{N} 1$ & $114.19(18)$ & $\mathrm{F} 4-\mathrm{B} 2-\mathrm{N} 4$ & $113.4(2)$ \\
\hline $\mathrm{F} 2-\mathrm{B} 1-\mathrm{N} 3$ & $109.65(19)$ & $\mathrm{F} 4-\mathrm{B} 2-\mathrm{N} 6$ & $110.52(18)$ \\
\hline $\mathrm{N} 1-\mathrm{B} 1-\mathrm{N} 3$ & $95.14(17)$ & $\mathrm{N} 4-\mathrm{B} 2-\mathrm{N} 6$ & $94.42(16)$ \\
\hline $\mathrm{O} 1-\mathrm{C} 10-\mathrm{C} 11-\mathrm{C} 12$ & $-178.6(2)$ & $\mathrm{O} 2-\mathrm{C} 22-\mathrm{C} 23-\mathrm{C} 24$ & $-179.2(2)$ \\
\hline $\mathrm{N} 1-\mathrm{C} 1-\mathrm{C} 4-\mathrm{C} 3$ & $0.1(3)$ & $\mathrm{N} 4-\mathrm{C} 13-\mathrm{C} 16-\mathrm{C} 15$ & $-0.5(3)$ \\
\hline $\mathrm{N} 1-\mathrm{C} 1-\mathrm{C} 4-\mathrm{C} 6$ & $-179.5(2)$ & $\mathrm{N} 4-\mathrm{C} 13-\mathrm{C} 16-\mathrm{C} 18$ & $179.0(2)$ \\
\hline $\mathrm{N} 1-\mathrm{C} 2-\mathrm{C} 3-\mathrm{C} 4$ & $0.7(3)$ & $\mathrm{N} 4-\mathrm{C} 14-\mathrm{C} 15-\mathrm{C} 16$ & $-0.2(3)$ \\
\hline $\mathrm{N} 2-\mathrm{N} 3-\mathrm{C} 7-\mathrm{C} 8$ & $8.1(3)$ & $\mathrm{N} 5-\mathrm{N} 6-\mathrm{C} 19-\mathrm{C} 20$ & $177.9(2)$ \\
\hline $\mathrm{N} 2-\mathrm{N} 3-\mathrm{C} 7-\mathrm{C} 12$ & $-171.1(2)$ & $\mathrm{N} 5-\mathrm{N} 6-\mathrm{C} 19-\mathrm{C} 24$ & $-0.4(3)$ \\
\hline $\mathrm{N} 2-\mathrm{N} 3-\mathrm{B} 1-\mathrm{F} 1$ & $117.8(2)$ & $\mathrm{N} 5-\mathrm{N} 6-\mathrm{B} 2-\mathrm{F} 3$ & $118.5(2)$ \\
\hline $\mathrm{N} 2-\mathrm{N} 3-\mathrm{B} 1-\mathrm{F} 2$ & $-119.2(2)$ & $\mathrm{N} 5-\mathrm{N} 6-\mathrm{B} 2-\mathrm{F} 4$ & $-116.8(2)$ \\
\hline $\mathrm{N} 2-\mathrm{N} 3-\mathrm{B} 1-\mathrm{N} 1$ & $-1.2(2)$ & $\mathrm{N} 5-\mathrm{N} 6-\mathrm{B} 2-\mathrm{N} 4$ & $0.2(2)$ \\
\hline $\mathrm{N} 2-\mathrm{C} 1-\mathrm{C} 4-\mathrm{C} 3$ & $178.7(3)$ & $\mathrm{N} 5-\mathrm{C} 13-\mathrm{C} 16-\mathrm{C} 15$ & $179.5(2)$ \\
\hline $\mathrm{N} 2-\mathrm{C} 1-\mathrm{C} 4-\mathrm{C} 6$ & $-0.8(4)$ & $\mathrm{N} 5-\mathrm{C} 13-\mathrm{C} 16-\mathrm{C} 18$ & $-1.0(4)$ \\
\hline $\mathrm{N} 3-\mathrm{N} 2-\mathrm{C} 1-\mathrm{N} 1$ & $-0.3(3)$ & N6-N5-C13-N4 & $0.2(3)$ \\
\hline $\mathrm{N} 3-\mathrm{N} 2-\mathrm{C} 1-\mathrm{C} 4$ & $-178.9(2)$ & $\mathrm{N} 6-\mathrm{N} 5-\mathrm{C} 13-\mathrm{C} 16$ & $-179.8(3)$ \\
\hline $\mathrm{N} 3-\mathrm{C} 7-\mathrm{C} 8-\mathrm{C} 9$ & $-177.5(2)$ & $\mathrm{N} 6-\mathrm{C} 19-\mathrm{C} 20-\mathrm{C} 21$ & $-178.7(2)$ \\
\hline $\mathrm{N} 3-\mathrm{C} 7-\mathrm{C} 12-\mathrm{C} 11$ & $178.5(2)$ & $\mathrm{N} 6-\mathrm{C} 19-\mathrm{C} 24-\mathrm{C} 23$ & $178.8(2)$ \\
\hline $\mathrm{C} 1-\mathrm{N} 1-\mathrm{C} 2-\mathrm{C} 3$ & $-0.6(2)$ & $\mathrm{C} 13-\mathrm{N} 4-\mathrm{C} 14-\mathrm{C} 15$ & $-0.1(3)$ \\
\hline $\mathrm{C} 1-\mathrm{N} 1-\mathrm{C} 2-\mathrm{C} 5$ & $178.5(2)$ & $\mathrm{C} 13-\mathrm{N} 4-\mathrm{C} 14-\mathrm{C} 17$ & $-179.9(2)$ \\
\hline $\mathrm{C} 1-\mathrm{N} 1-\mathrm{B} 1-\mathrm{F} 1$ & $-116.2(2)$ & $\mathrm{C} 13-\mathrm{N} 4-\mathrm{B} 2-\mathrm{F} 3$ & $-116.9(2)$ \\
\hline $\mathrm{C} 1-\mathrm{N} 1-\mathrm{B} 1-\mathrm{F} 2$ & $115.2(2)$ & $\mathrm{C} 13-\mathrm{N} 4-\mathrm{B} 2-\mathrm{F} 4$ & $114.5(2)$ \\
\hline $\mathrm{C} 1-\mathrm{N} 1-\mathrm{B} 1-\mathrm{N} 3$ & $1.0(2)$ & $\mathrm{C} 13-\mathrm{N} 4-\mathrm{B} 2-\mathrm{N} 6$ & $-0.1(2)$ \\
\hline $\mathrm{C} 1-\mathrm{N} 2-\mathrm{N} 3-\mathrm{C} 7$ & $-177.34(19)$ & $\mathrm{C} 13-\mathrm{N} 5-\mathrm{N} 6-\mathrm{C} 19$ & $-179.84(19)$ \\
\hline $\mathrm{C} 1-\mathrm{N} 2-\mathrm{N} 3-\mathrm{B} 1$ & $1.0(2)$ & $\mathrm{C} 13-\mathrm{N} 5-\mathrm{N} 6-\mathrm{B} 2$ & $-0.3(2)$ \\
\hline $\mathrm{C} 2-\mathrm{N} 1-\mathrm{C} 1-\mathrm{N} 2$ & $-178.58(19)$ & $\mathrm{C} 14-\mathrm{N} 4-\mathrm{C} 13-\mathrm{N} 5$ & $-179.60(19)$ \\
\hline $\mathrm{C} 2-\mathrm{N} 1-\mathrm{C} 1-\mathrm{C} 4$ & $0.4(3)$ & $\mathrm{C} 14-\mathrm{N} 4-\mathrm{C} 13-\mathrm{C} 16$ & $0.4(3)$ \\
\hline $\mathrm{C} 2-\mathrm{N} 1-\mathrm{B} 1-\mathrm{F} 1$ & $60.6(4)$ & $\mathrm{C} 14-\mathrm{N} 4-\mathrm{B} 2-\mathrm{F} 3$ & $62.3(4)$ \\
\hline $\mathrm{C} 2-\mathrm{N} 1-\mathrm{B} 1-\mathrm{F} 2$ & $-68.0(4)$ & $\mathrm{C} 14-\mathrm{N} 4-\mathrm{B} 2-\mathrm{F} 4$ & $-66.2(4)$ \\
\hline $\mathrm{C} 2-\mathrm{N} 1-\mathrm{B} 1-\mathrm{N} 3$ & $177.8(3)$ & $\mathrm{C} 14-\mathrm{N} 4-\mathrm{B} 2-\mathrm{N} 6$ & $179.2(3)$ \\
\hline $\mathrm{C} 2-\mathrm{C} 3-\mathrm{C} 4-\mathrm{C} 1$ & $-0.4(3)$ & $\mathrm{C} 14-\mathrm{C} 15-\mathrm{C} 16-\mathrm{C} 13$ & $0.5(3)$ \\
\hline $\mathrm{C} 2-\mathrm{C} 3-\mathrm{C} 4-\mathrm{C} 6$ & $179.1(2)$ & $\mathrm{C} 14-\mathrm{C} 15-\mathrm{C} 16-\mathrm{C} 18$ & $-179.0(2)$ \\
\hline $\mathrm{C} 5-\mathrm{C} 2-\mathrm{C} 3-\mathrm{C} 4$ & $-178.4(2)$ & $\mathrm{C} 17-\mathrm{C} 14-\mathrm{C} 15-\mathrm{C} 16$ & $179.6(2)$ \\
\hline $\mathrm{C} 7-\mathrm{N} 3-\mathrm{B} 1-\mathrm{F} 1$ & $-64.0(3)$ & $\mathrm{C} 19-\mathrm{N} 6-\mathrm{B} 2-\mathrm{F} 3$ & $-62.0(3)$ \\
\hline
\end{tabular}




$\begin{array}{llll}\mathrm{C} 7-\mathrm{N} 3-\mathrm{B} 1-\mathrm{F} 2 & 59.0(3) & \mathrm{C} 19-\mathrm{N} 6-\mathrm{B} 2-\mathrm{F} 4 & 62.7(3) \\ \mathrm{C} 7-\mathrm{N} 3-\mathrm{B} 1-\mathrm{N} 1 & 176.9(2) & \mathrm{C} 19-\mathrm{N} 6-\mathrm{B} 2-\mathrm{N} 4 & 179.7(2) \\ \mathrm{C} 7-\mathrm{C} 8-\mathrm{C} 9-\mathrm{C} 10 & -1.6(4) & \mathrm{C} 19-\mathrm{C} 20-\mathrm{C} 21-\mathrm{C} 22 & -0.4(3) \\ \mathrm{C} 8-\mathrm{C} 7-\mathrm{C} 12-\mathrm{C} 11 & -0.7(3) & \mathrm{C} 20-\mathrm{C} 19-\mathrm{C} 24-\mathrm{C} 23 & 0.5(3) \\ \mathrm{C} 8-\mathrm{C} 9-\mathrm{C} 10-\mathrm{O} 1 & 179.7(2) & \mathrm{C} 20-\mathrm{C} 21-\mathrm{C} 22-\mathrm{O} 2 & 179.4(2) \\ \mathrm{C} 8-\mathrm{C} 9-\mathrm{C} 10-\mathrm{C} 11 & 0.3(4) & \mathrm{C} 20-\mathrm{C} 21-\mathrm{C} 22-\mathrm{C} 23 & 1.1(3) \\ \mathrm{C} 9-\mathrm{C} 10-\mathrm{C} 11-\mathrm{C} 12 & 0.8(3) & \mathrm{C} 21-\mathrm{C} 22-\mathrm{C} 23-\mathrm{C} 24 & -1.0(3) \\ \mathrm{C} 10-\mathrm{C} 11-\mathrm{C} 12-\mathrm{C} 7 & -0.5(3) & \mathrm{C} 22-\mathrm{C} 23-\mathrm{C} 24-\mathrm{C} 19 & 0.1(3) \\ \mathrm{C} 12-\mathrm{C} 7-\mathrm{C} 8-\mathrm{C} 9 & 1.8(4) & \mathrm{C} 24-\mathrm{C} 19-\mathrm{C} 20-\mathrm{C} 21 & -0.4(3) \\ \mathrm{B} 1-\mathrm{N} 1-\mathrm{C} 1-\mathrm{N} 2 & -0.6(3) & \mathrm{B} 2-\mathrm{N} 4-\mathrm{C} 13-\mathrm{N} 5 & -0.1(3) \\ \mathrm{B} 1-\mathrm{N} 1-\mathrm{C} 1-\mathrm{C} 4 & 178.34(19) & \mathrm{B} 2-\mathrm{N} 4-\mathrm{C} 13-\mathrm{C} 16 & 179.92(19) \\ \mathrm{B} 1-\mathrm{N} 1-\mathrm{C} 2-\mathrm{C} 3 & -177.4(3) & \mathrm{B} 2-\mathrm{N} 4-\mathrm{C} 14-\mathrm{C} 15 & -179.3(3) \\ \mathrm{B} 1-\mathrm{N} 1-\mathrm{C} 2-\mathrm{C} 5 & 1.7(4) & \mathrm{B} 2-\mathrm{N} 4-\mathrm{C} 14-\mathrm{C} 17 & 0.9(5) \\ \mathrm{B} 1-\mathrm{N} 3-\mathrm{C} 7-\mathrm{C} 8 & -170.0(2) & \mathrm{B} 2-\mathrm{N} 6-\mathrm{C} 19-\mathrm{C} 20 & -1.6(3) \\ \mathrm{B} 1-\mathrm{N} 3-\mathrm{C} 7-\mathrm{C} 12 & 10.8(3) & \mathrm{B} 2-\mathrm{N} 6-\mathrm{C} 19-\mathrm{C} 24 & -179.8(2) \\ \end{array}$

Hydrogen-bond geometry $\left(A,{ }^{\circ}\right)$

$\mathrm{Cg} 2$ and $\mathrm{Cg} 6$ are the centroids of the $\mathrm{N} 4 / \mathrm{C} 13-\mathrm{C} 16$ and $\mathrm{N} 1 / \mathrm{C} 1-\mathrm{C} 4$ rings, respectively.

\begin{tabular}{lllll}
\hline$D-\mathrm{H} \cdots A$ & $D-\mathrm{H}$ & $\mathrm{H} \cdots A$ & $D \cdots A$ & $D-\mathrm{H} \cdots A$ \\
\hline $\mathrm{O} 1-\mathrm{H} 1 \cdots \mathrm{O} 2$ & 0.82 & 1.98 & $2.797(2)$ & 178 \\
$\mathrm{O} 2-\mathrm{H} 2 \cdots \mathrm{F} 2^{\mathrm{i}}$ & 0.82 & 2.06 & $2.812(2)$ & 152 \\
$\mathrm{C} 3-\mathrm{H} 3 \cdots C g 1^{\mathrm{ii}}$ & 0.93 & 2.62 & $3.501(2)$ & 158 \\
$\mathrm{C} 15-\mathrm{H} 15 \cdots C g 2^{\mathrm{iii}}$ & 0.93 & 2.63 & $3.506(2)$ & 157 \\
\hline
\end{tabular}

Symmetry codes: (i) $x-1, y, z$; (ii) $-x+3 / 2, y+1 / 2,-z+3 / 2$; (iii) $-x+1 / 2, y-1 / 2,-z+3 / 2$. 